\title{
Heterogeneous Forecasts and Aggregate Dynamics
}

\author{
Antulio N. Bomfim* \\ Division of Monetary Affairs, Federal Reserve Board, Washington, DC, 20551, USA
}

January 19,2000

\begin{abstract}
Motivated by issues raised in both the finance and economics literatures, I construct a dynamic general equilibrium model where agents use differing degrees of sophistication when forecasting future economic conditions. All agents solve standard dynamic optimization problems and face strategic complementarity in production, but some solve their inference problems based on simple forecasting rules of thumb. Assuming a hierarchical information structure similar to the one in Townsend's (1983) model of informationally dispersed markets, I show that even a minority of rule-of-thumb forecasters can have a significant effect on the aggregate properties of the economy. For instance, as agents try to forecast each others' behavior they effectively strengthen the internal propagation mechanism of the economy. The quantitative results are obtained by calibrating the model and running a battery of sensitivity tests on key parameters. The analysis highlights the role of strategic complementarity in the heterogeneous expectations literature and quantifies many qualitative claims about the aggregate implications of expectational heterogeneity.
\end{abstract}

JEL Classification: E32, D82, C61

Keywords: expectations, business cycles, strategic complementarity, propagation, bounded rationality

* E-mail: abomfim@frb.gov, Fax: (202) 452-2301, Tel.: (202) 736-5619.

I received valuable comments from an anonymous referee, Robert King, Michael Binder, Satyajit Chatterjee, Russell Cooper, Frank Diebold, John Haltiwanger, Michael Waldman, and seminar participants at the 1997 North American Winter Meetings of the Econometric Society. Steve Sumner provided excellent research assistance in earlier stages of this project. The opinions expressed here are not necessarily shared by the Board of Governors of the Federal Reserve System or any other members of its staff. 


\section{Introduction}

Although the rational expectations assumption is a standard fixture of most work in modern macroeconomics and finance, a growing number of studies has raised concerns about its theoretical and empirical implications. For instance, Frydman and Phelps (1983), Board (1994), and Arthur (1994) argue that human rationality is bounded, and thus the rational expectations assumption imposes extreme informational and computational requirements on agents (see also Sargent, 1993). In addition, other researchers base their reservations on concerns about the observable characteristics of rational expectations equilibria. For example, De Long, Schleifer, Summers, and Waldmann (1990) constructed a model in which behavior based on irrational noise trading helps explain a number of observed phenomena in financial markets, such as the excess volatility of asset prices and the equity premium puzzle. Also in the finance literature, Roll (1997) mentions incomplete (bounded) rationality as a possible explanation for the observation of large trade volumes in debt markets. Whereas these reservations have led some economists to discard the rational expectations hypothesis altogether, others have sought to reconsider it in the context of environments with less demanding informational assumptions and more plausible observable implications. Lucas (1975) and Townsend (1983) were among the first to take up this line of inquiry. Townsend, in particular, analyzed a model where agents form heterogeneous expectations because their forecasts are conditioned on different subsets of the relevant data. He showed that, as agents attempt to "forecast the forecast of others," the economy converges to a rational expectations equilibrium.

Subsequently, the work of Haltiwanger and Waldman $(1985,1989)$ brought a new perspective to the analysis of forecast heterogeneity. Rather than working with dynamic models with no externalities (the Lucas-Townsend approach), Haltiwanger and Waldman analyzed environments that allowed for strategic complementarity. ${ }^{1}$ In addition, instead of focusing on agents with different access to the data, they built models where forecast heterogeneity arises because some agents may use more sophisticated forecasting methods than others, an idea that is formalized in the work of Evans and Ramey (1992) and Sethi and Franke (1995). Unlike the results obtained by papers in the Lucas-Townsend tradition, the class of simple, static models analyzed by Haltiwanger and Waldman gave rise to environments where forecast heterogeneity did cast some doubt on the aggregate implications of the rational expectations hypothesis. They showed that, with strategic complemen-

\footnotetext{
${ }^{1}$ Cooper and John (1988) define strategic complementarity and discuss its implications for macroeconomics. For the purpose of my paper, strategic complementarity involves a situation where an individual's output decision is increasing in the level of aggregate output.
} 
tarity, less sophisticated forecasters may have a sizable effect on the evolution of aggregate output, effectively driving the economy away from its pure rational expectations equilibrium.

In this paper I introduce and solve a model that brings together important issues stemming from both the Lucas-Townsend and Haltiwanger-Waldman approaches to analyzing heterogeneousexpectations models. Capturing the key insights of Haltiwanger and Waldman, my analysis allows for strategic complementarity and heterogeneous forecasting rules. However, to bring the discussion more into the current stage of macroeconomic thought-which emphasizes dynamic rather than static frameworks - I extend the Haltiwanger-Waldman analysis to a richer dynamic general equilibrium model, which I solve with a methodology that is very close in spirit to Townsend's.

In choosing a specific dynamic modeling framework, I opted for the class of models in the real business cycle $(\mathrm{RBC})$ tradition. The advantages of this choice are two-fold. First, the RBC framework is well understood by the profession, and the results I obtain under forecast heterogeneity and strategic complementarity can be directly and quantitatively compared to those generated by standard RBC models. Second, by introducing forecast heterogeneity into the RBC framework, I am able to address a recurring theme in the literature: the weak internal propagation mechanism that underlies many equilibrium business cycle models (Cogley and Nason, 1995).

The paper's main results and methodology can be summarized as follows. With a sufficiently strong degree of strategic complementarity, I show that even if only a small subset of agents forecasts according to a simple but reasonable rule of thumb, aggregate output exhibits more persistence than warranted by either (i) the degree of serial correlation in the productivity shock process, or (ii) the share of rule-of-thumb forecasters in the total population. More to the point, because agents engage in forecasting each others' forecasts, rule-of-thumb forecasters have a quantitatively important impact on the serial correlation properties of the business cycle. The results are obtained by calibrating all standard RBC parameters and then running a battery of sensitivity tests on the forecast-heterogeneity and strategic-complementarity parameters. The sensitivity tests highlight the role of strategic complementarity in the forecast-heterogeneity debate and precisely quantify the qualitative claims made by Haltiwanger and Waldman and others.

\section{The Model}

Apart from the issue of heterogeneity, the model is very close to that of Baxter and King (1991). 


\subsection{Technology and Preferences}

Strategic Complementarity and the Production Function. Individual output is a function not only of inputs and a productivity shock, but also of an index of per capita aggregate output. This index is a geometric average of the per capita output decisions of two types of agents:

$$
Y_{t}=Y_{R, t}^{\theta_{R}} Y_{S, t}^{\left(1-\theta_{R}\right)}
$$

where $\theta_{R}$ is the population share of rule-of-thumb forecasters, and $Y_{R, t}$ is their per capita output decision. (Rule-of-Thumb forecasting is discussed in the next section.) The $S$ subscript denotes variables pertaining to the sophisticated forecasters.

An agent of type $i$ faces the production function,

$$
y_{i, t}=\exp \left(A_{t}\right) F\left(k_{i, t-1}, n_{i, t}\right) Y_{t}^{\phi} \quad i=R, S
$$

where $y_{i, t}$ denotes individual output, and $k_{i, t-1}$ and $n_{i, t}$ are the capital and labor inputs. $A_{t}$ is assumed to capture stochastic shifts in productivity; it follows a first-order autoregression with autocorrelation coefficient $\rho$ and innovation $a_{t}$, which is normally distributed with zero mean. $F($.) is a Cobb-Douglas production function, $F\left(k_{i, t-1}, n_{i, t}\right) \equiv k_{i, t-1}^{\theta_{k}} n_{i, t}^{\theta_{n}}$, which is homogeneous of degree 1. The $\phi$ parameter in equation (2) embodies the complementarity assumption; it determines the extent to which individual output, $y_{i, t}$, depends on aggregate output, $Y_{t}$. $(0 \leq \phi<1)$

Evolution of the Capital Stock. Output not consumed constitutes gross investment, $i_{i, t}$. With $k_{i, t}$ representing the capital stock at the end of period $t$, and assuming that this stock depreciates at the rate $\delta, 0 \leq \delta<1$,

$$
k_{i, t}=(1-\delta) k_{i, t-1}+i_{i, t}
$$

Preferences. Preferences are homogeneous throughout the economy. The momentary utility function of a representative agent is defined in terms of consumption $\left(c_{i, t}\right)$ and leisure $\left(l_{i, t}\right)$,

$$
u\left(c_{i, t}, l_{i, t}\right)=\log \left(c_{i, t}\right)+\theta_{l} \log \left(l_{i, t}\right)
$$

where leisure is expressed as a proportion of the unit time endowment. 


\subsection{Individual Behavior}

The economy is populated by utility-maximizing, infinitely-lived, forward-looking agents who discount the future at the rate $\beta$. As in Townsend (1983), we can think of individual behavior as the outcome of two separate problems: dynamic optimization and inference. The solution to the first problem yields the perfect-foresight equilibrium laws of motion of all choice variables, which express the evolution of these variables as a function of past, current, and future states of the economy. The assumption of heterogeneous expectations amounts to saying that agents use the same mechanism to solve their dynamic optimization problem, but not their inference problem.

The Dynamic Optimization Problem. Given equations (1) through (4) and the usual time and goods constraints, the dynamic optimization problem reduces to solving

$$
\max \sum_{t=0}^{\infty} \beta^{t}\left\{u\left(c_{i, t}, 1-n_{i, t}\right)+\lambda_{t}\left[\exp \left(A_{t}\right) F(.) Y_{t}^{\phi}-c_{i, t}-k_{i, t}+(1-\delta) k_{i, t-1}\right]\right\}
$$

subject to $k_{i,-1}$, the transversality condition $\lim _{t \rightarrow \infty} \beta^{t} \lambda_{t} k_{i, t}=0$, and for given $\left\{A_{t}, Y_{t}\right\}_{t=0}^{\infty}$. $\left(\lambda_{t}\right.$ is the discounted Lagrange multiplier relevant for time $t$.)

The perfect-foresight equilibrium paths of consumption, investment, and labor effort are given by the solution to the system of Euler equations that corresponds to (5). Given that generally there is no closed-form solution to this system, my focus is on an approximate solution, obtainable by loglinearizing the system around its steady state. ${ }^{2}$ After imposing a symmetry condition that says that individuals who rely on the same forecasting mechanism make identical decisions in equilibrium, the (approximate) equilibrium laws of motion take the form

$$
x_{i, t}=\Pi_{i, k} \hat{K}_{i, t-1}+\Pi_{i, \lambda} \tilde{\lambda}_{i, t}+\Pi_{i, e} e_{i, t}, \quad i, j=S, R ; j \neq i
$$

where $x_{i, t} \equiv\left[\hat{N}_{i, t}, \hat{K}_{i, t}, \hat{C}_{i, t}\right]^{\prime}, e_{i, t} \equiv\left[A_{t}, \hat{Y}_{j, t}\right]^{\prime}$, and $\tilde{\lambda}_{i, t} \equiv \sum_{h=0}^{\infty} \mu_{i}^{-h}\left(F_{i, 1} e_{i, t+h+1}+F_{i, 2} e_{i, t+h}\right)$.

A "caret" over a symbol denotes that the variable is expressed in percentage deviations from the steady state (e.g., $\hat{Y}_{i, t} \equiv \log \left(Y_{i, t} / \bar{Y}_{i}\right)$ ), and upper-case letters denote per-capita variables. The matrices $\Pi_{i}$ and $F_{i}$, as well as the $\mu_{i}$ parameter, are functions of various steady-state properties of the model, such as the steady-state capital-output ratio and the labor share of total income.

The Inference Problem. To fully characterize individual behavior, I still need to explicitly address the issues of uncertainty and expectational formation. For each period $t$, I assume that all agents

\footnotetext{
${ }^{2}$ The log-linear approximation method used here is described in detail in King, Plosser, and Rebelo (1990).
} 
follow a two-stage decision process. ${ }^{3}$ At the beginning of the period, the first stage takes place: agents make their labor supply and capital accumulation decisions before being able to observe the current value of the productivity shifter, $A_{t}$, or the current output decision of the other agents in the economy. The factor-allocation decision rules take the form:

$$
z_{i, t}=\pi_{i, k} \hat{K}_{i, t-1}+\pi_{i, \lambda} E^{(i)}\left[\tilde{\lambda}_{i, t} \mid \Omega_{t-1}\right]+\pi_{i, e} E^{(i)}\left[e_{i, t} \mid \Omega_{t-1}\right]
$$

where $z_{i, t} \equiv\left[\hat{N}_{i, t}, \hat{K}_{i, t}\right]^{\prime}$, and the $\pi_{i}$ parameters correspond to the appropriate elements of the $\Pi_{i}$ matrices from equation (6). $\Omega_{t-1}$ is the information set available at the beginning of period $t$; it contains the whole history of the economy up to period $t-1 . E^{(i)}\left[. \mid \Omega_{t-1}\right]$ denotes the expectation of a type $i$ agent conditioned on $\Omega_{t-1}$.

Given last period's capital stock and this period's factor-allocation decisions, production and capital accumulation take place, and the agents move on to the second and last stage of their decision making process. Assuming that both sophisticated and rule-of-thumb forecasters observe each others' output as soon as production occurs, each agent can use its knowledge of the production function to deduce the current value of the productivity shifter $\left(A_{t}\right)$. Therefore, the consumption decision is based on a larger information set, $\Omega_{0, t} \equiv\left\{\Omega_{t-1}, A_{t}, Y_{S, t}, Y_{R, t}\right\}$.

Equation (7) makes explicit two points advanced earlier in this paper. First, agents of different types are informationally linked: To generate their own decision rules they must forecast the behavior of the other agents in the economy-recall that $\hat{Y}_{j, t}$ is an element of $e_{i, t}$. Second, equation (7) highlights the channel through which agents' expectations affect their behavior: The different expectational rules embedded in $E^{(S)}$ and $E^{(R)}$ can lead to potentially different responses to the same fundamental shocks.

\section{Expectational Heterogeneity}

Sophisticated agents make use of full knowledge of the structure of the model, including the expectational behavior of rule-of-thumb agents. For any given variable $\psi_{t}$, their expectations can be formally defined as $E^{(S)}\left[\psi_{t} \mid \Omega_{t-1}\right]=E\left[\psi_{t} \mid \Omega_{t-1}\right]$, which is the mathematical expectation of $\psi_{t}$ conditioned on the information set $\Omega_{t-1}$ and the true structure of the entire model.

\footnotetext{
${ }^{3}$ Kydland and Prescott (1982) assume a similar information structure.
} 


\subsection{Rule-of-Thumb Forecasting}

Three criteria guided the specification of an illustrative forecasting model for rule-of-thumb agents. First, to capture the concerns of the costly-implementation literature - e.g. Evans and Ramey (1992) and Board (1994) - the rule must be simple to implement. Second, the forecasting rule should generate "reasonable" forecasts, i.e., it should be consistent with well-known characteristics of the economy. Finally, the expectational model must not be at odds with the agents' ability to solve their dynamic optimization problem. In other words, when solving their dynamic optimization and inference problems, rule-of-thumb agents must rely on a single, consistent pool of information about the behavior of the economy.

According to (7), a type $i$ agent must forecast current and future movements in total factor productivity $\left(A_{t}\right)$ and the per capita output of the other agents in the economy $\left(\hat{Y}_{j, t}\right)$. For illustrative purposes, suppose that rule-of-thumb agents rely on an autoregressive forecasting model:

$$
\begin{aligned}
E^{(R)}\left[A_{t+h} \mid \Omega_{t-1}\right] & =\alpha^{h+1} A_{t-1} \\
E^{(R)}\left[\hat{Y}_{S, t+h} \mid \Omega_{t-1}\right] & =\alpha^{h+1} \hat{Y}_{S, t-1}
\end{aligned}
$$

To see how the above forecasting structure fares with the three criteria listed above, note that, first, especially for $\alpha$ close or equal to $\rho$, the forecasting model in equation (8) is not only simple, reasonable, and consistent with their ability to solve their dynamic optimization problem, but also, for $\alpha=\rho$, perfectly rational. Second, while it is obvious that (9) is only an approximation to the true process governing the evolution of $\hat{Y}_{S, t}$, it captures a well-known feature of traditional RBC models: the fact that output persistence is tightly linked to the degree of serial correlation in the productivity shock series. Thus, rather than taking the time and resources to compute a fully model-consistent forecast for $\hat{Y}_{S, t}$, a practice that would considerably complicate the solution to the model, rule-ofthumb agents use the simple model given by (9). Finally, note that the ability of rule-of-thumb agents to solve their dynamic optimization problem with the same of level of sophistication as the other agents is not inconsistent with the relatively unsophisticated methods they use in solving their inference problem. The solution to the dynamic optimization problem requires structural information only about one's own constraints and opportunities; by assumption, both types of agents use this information. However, to solve their inference problem in a way consistent with the rational expectations hypothesis, individuals also need complete structural information on the constraints and opportunities facing the other agents in the economy; by assumption, sophisticated 
agents process this information, rule-of-thumb forecasters do not.

In several aspects, the expectational assumptions made so far are similar to Townsend's (1983) description of a hierarchical informational structure. Sophisticated forecasters are placed higher in the hierarchy: In addition to the information that enables them to solve their dynamic optimization problem, they also know the precise nature of the dynamic optimization and inference problems being solved by the rule-of-thumb agents. Thus, their forecasts incorporate structural information about the whole economy. In contrast, the structural information embodied in the forecasting behavior of the rule-of-thumb forecasters is self-contained: They use information about their own constraints and opportunity sets, but not those of sophisticated agents.

\subsection{Decision Rules under Heterogeneous Expectations}

Given the hierarchical information structure, the model can be solved sequentially in two steps. Starting at the bottom of the hierarchy, I first derive the decision rules of rule-of-thumb agents and then use the results to compute the more complicated decision rules of the sophisticated forecasters. Rule-of-Thumb Agents. The factor-allocation decision rule of rule-of-thumb agents,

$$
z_{R, t}=\pi_{R, k} \hat{K}_{R, t-1}+\pi_{R, A} A_{t-1}+\pi_{R, Y} \hat{Y}_{S, t-1}
$$

is obtained by substituting their expectations formulae - equations (8) and (9) - into the perfectforesight equilibrium law of motion of $z_{R, t}$-equation (7). Given the factor-allocation decisions, production and consumption take place.

Sophisticated Agents. Like the rule-of-thumb forecasters, sophisticated agents make their factorallocation and consumption decisions in two states and subject to the same information sets, $\Omega_{t-1}$ and $\Omega_{0, t}$. Thus, their factor-allocation decisions are made before they can observe either the output decision of the other agents or the current state of productivity. However, to form expectations about $Y_{R, t}$, sophisticated agents look not only at their own dynamic optimization problem, but also at the decisions and forecasting models used by rule-of-thumb agents. This information is subsumed in equations (6), through (10), which can be grouped together with the appropriate Euler equations to form a two-sided matrix difference equation that can be solved for the decision variables.

Aggregation. Having outlined above how different types of agents go about solving their respective utility maximization problems, my ultimate interest lies on the analysis of the dynamics of the economy as a whole. As it turns out, the evolution of aggregate variables can be easily derived 
from the individual decision rules computed above - see equation (1).

\section{Quantitative Business-Cycle Analysis}

To assess whether the introduction of forecast heterogeneity affects the dynamic properties of a realbusiness-cycle economy, I run what Kydland and Prescott (1996) call a computational experiment (see also King, 1995). The final steps of such an experiment involve calibrating the model to allow for meaningful quantitative analysis and then running the experiment itself.

\subsection{Model Calibration}

With the exception of the forecast-heterogeneity and complementarity parameters, $\left[\theta_{R}, \alpha, \phi\right]$, all model parameters are calibrated as in King, Plosser, and Rebelo (1988).

Strategic complementarity. The calibration of the strategic complementarity parameter $(\phi)$ is guided by the empirical work of Baxter and King (1991), Caballero and Lyons (1989, 1992), Cooper and Haltiwanger (1996), and Basu and Fernald (1995, 1997). However, even a casual look at these papers reveals a very wide range of estimates for $\phi$. For instance, Baxter and King and Caballero and Lyons report estimates that range from from 0.1 to 0.49 , and, while the results obtained by Basu and Fernald point towards the lower range of these estimates, Cooper and Haltiwanger report even larger estimates. For the purposes of this paper, rather than making a case for any particular estimate of $\phi$, I run my experiments using a range of values for $\phi$ that is consistent with the diverse

findings of the empirical literature and then trace the consequences of these different estimates for the aggregate effects of expectational heterogeneity.

Serial correlation and volatility of technology shocks. Two parameters that do not affect the steadystate properties of the model, but play a crucial role in aggregate fluctuations, are the innovation variance and the autoregressive coefficient of $\hat{A}_{t},\left(\sigma_{a}^{2}\right.$ and $\rho$, respectively). To calibrate $\sigma_{a}^{2}$, I simply set it to a value that makes the model's output variance equal to its empirical counterpart. Nonetheless, as long as my focus rests on the propagation mechanism, my results are invariant to the particular parameterization of $\sigma_{a}^{2}$.

The parameterization of $\rho$ is designed to highlight a recurring weakness of the standard RBC model with homogeneous expectations: the lack of a quantitatively important internal propagation mechanism (Cogley and Nason, 1995). As is well known, in order to be able to mimic the degree of serial correlation in the data, most RBC models require near-unit root processes for $A_{t}$, effectively 
implying that persistence is exogenously imposed on the system, rather than explained by it. To isolate the role of expectational heterogeneity in the persistent generation process, I start by setting $\rho$ at 0.5 , about half the usual parameterization adopted in standard RBC models.

Rule-of-Thumb Forecasting. If there were some precision concerns surrounding the available estimates of the strategic complementarity parameter, we are hard pressed to find any estimates, however imprecise, of the expectational parameters of the model $\left(\theta_{R}\right.$ and $\left.\alpha\right)$. Thus, I shall treat $\theta_{R}$ and $\alpha$ as semi-free parameters and experiment with a wide range of values for each of them. Accordingly, I view my results as a mapping from the magnitudes of the $\phi, \theta_{R}$ and $\alpha$ parameters to the properties of the artificial time series generated by the model. The rest of this section provides a sensitivity analysis that reflects this mapping. ${ }^{4}$

\subsection{Expectational Heterogeneity and the Propagation Mechanism}

I start by examining an economy without strategic complementarity $(\phi=0)$. Table 1-A summarizes the autocorrelation function of aggregate output under alternative values of the expectational parameters. In particular, the table reports the results of computational experiments that explore different degrees of rule-of-thumb agents' misperception about the persistence of technological shocks - for $\rho=0.5, \alpha$ is set to $0.3,0.5$, and 0.7 -and different shares of rule-of-thumb agents in the total population $-\theta_{R}$ varies from 0 to 0.9 .

Three main results are evident Table 1-A. First, the impact of rule-of-thumb forecasters on the persistence of output is potentially negligible, even if we allow these agents to make up the vast majority of the population $\left(\theta_{R}=0.9\right)$. Second, though quantitatively small, the particular expectational model used by rule-of-thumb forecasters has a noteworthy property: Their misperceptions about the persistence of the technological shock are reflected in the actual serial correlation of output: Whenever they expect the shocks to be more [less] persistent than warranted by the data generating process, aggregate output ends up slightly more [less] persistent than otherwise. Third, when there are no misperceptions about the size of $\rho$, the heterogeneous expectations model essentially reduces to a standard $\mathrm{RBC}$ model. Taken together, the results suggest that the inclusion of unsophisticated forecasters in the RBC model without strategic complementarity produced quantitatively insignificantly effects: With $\phi$ set to zero, small deviations from the rational expectations assumption produce only small deviations from the standard RBC results.

\footnotetext{
${ }^{4} \mathrm{I}$ am grateful to an anonymous referee for suggesting this course of inquiry.
} 
Expectational Heterogeneity under Strategic Complementarity. Table 1-B summarizes the results of computational experiments identical to the ones described above, except that now I set the strategic complementarity parameter to 0.49. The results stand in stark contrast to the ones reported in Table 1-A: Even when the rule-of-thumb forecasters represent only a minority of the population, the serial correlation properties of output may be affected in a quantitatively important way. For instance, if these agents over-estimate the persistence of the technological shock $(\alpha=0.70$, $\rho=0.50$ ), aggregate output becomes noticeably more persistent than in the case of purely rational expectations even with $\theta_{R}=0.30$. Thus, unlike the case of no strategic complementarity, even small deviations from the rational expectations hypothesis can lead to significant deviations from standard RBC results when external returns are high .

Tables 1-A and 1-B highlight an important feature of the model. Note that, for given $\alpha$ and $\phi$, the effect of rule-of-thumb forecasting on the persistence of aggregate output does not generally monotonically increase with $\theta_{R}$. For instance, for $\alpha=0.70$, Table 1-B shows that output actually becomes slightly less persistent as the share of rule-of-thumb forecasters in the population increases from 0.60 to 0.90 . This finding has important implications for the study of the aggregate effects of expectational heterogeneity. What it says is that persistence is not simply being exogenously generated as a result of the introduction of rule-of-thumb agents. In addition to this exogenous factor, the expectations-induced propagation mechanism featured in this paper has an important endogenous component: Strategic complementarity strengthens the informational links between the two types of agents, which in turn leads the sophisticated forecasters to incorporate into their decision making process the autoregressive nature of the forecasting models used by the ruleof-thumb agents (see subsection 4.4 for additional discussion of the interactions between the two types of agents). Therefore, as the population share of sophisticated agents decreases, the aggregate implications of their optimal responses to rule-of-thumb forecasting is dampened, and this explains why the relative effect of rule-of-thumb forecasting eventually decreases at very high values of $\theta_{R}$.

Thus far I have reported on the effects of rule-of-thumb forecasting under what might be called two polar assumptions about the magnitude of the strategic complementarity parameter: the zero lower bound featured in most RBC models and 0.49, which is at the high end of the range of estimates discussed in the previous section. A question of interest is what happens at intermediate values of $\phi$. Indeed, I ran the computational experiments reported in Table 1-B for all point estimates of $\phi$ obtained by Baxter and King (1991) and Caballero and Lyons (1992) $-\phi=0.10,0.32$, 0.45, and 0.49. (I did not include the higher estimates reported by Cooper and Haltiwanger, 1996, as they would violate the model's stability conditions.) The impact of the type of expectational 
heterogeneity examined in this paper is still quite sizable for $\phi=0.45$, but the results are not as dramatic for the two lower values of the external returns parameter, $\phi=0.10$ and $\phi=0.32$. These results highlight the fact that more precise estimates of the actual degree of strategic complementarity are crucial for a more definitive assessment of the aggregate (quantitative) implications of forecast heterogeneity.

\subsection{Model Evaluation}

Although I cannot fully calibrate all parameters of the model and compare its time series properties with selected moments of the data, it is still useful to verify whether a plausible parameterization of the heterogeneous-expectation RBC model with strategic complementarity can make it roughly consistent with the data.

Table 2-A, extracted from King et al. (1988), summarizes the selected moments of the U.S. data that the model will try to match. The corresponding model moments are shown in Table 2-B. The results reported in this table are obtained by assuming a relatively high degree of complementarity $(\phi=0.50)$, while potentially allowing for only a limited role for rule-of-thumb forecasting $\left(\theta_{R}=\right.$ 0.30). To highlight the internal propagation mechanism coming from heterogeneous expectations under strategic complementarity, I arbitrarily set the persistence parameter $(\rho)$ at 0.70 , lower than what a standard RBC model would require to capture the serial correlation observed in the data. Assuming no misperceptions from the part of rule-of-thumb agents $(\alpha=\rho)$, the model replicates well the serial correlation of the data, especially for consumption and output. The observed relative volatilities of output, consumption, and investment are also largely captured by the model, though consumption and investment are a bit too volatile and hours do not vary as much as in the data.

To compare the internal propagation of the model with the standard RBC framework, Table 2-C shows the same selected moments shown in Table 2-B, but now the complementarity and expectational parameters are both set to zero. As expected, without strong serial correlation in the shocks, the standard RBC model fails to capture the observed serial correlation of output.

\subsection{Disaggregated Dynamics}

In a statistical (mean-squared-error) sense, the forecasts formed by rule-of-thumb agents are less efficient than the model-consistent expectations of the sophisticated forecasters. The goal of this subsection is to informally check whether the rule-of-thumb agents' reliance on statistically suboptimal forecasts makes them behave substantially different from the sophisticated forecasters. 
Consider, for example, that different agents may face different costs of forming sophisticated forecasts. If the behavioral differences between sophisticated and rule-of-thumb forecasters are large, either these (unspecified) costs are very sizable or there is something inherently irrational about rule-of-thumb forecasting. On the other hand, if behavioral differences between the two types of agents are small, then even small costs associated with forming sophisticated forecasts could potentially motivate forecasting based on rules of thumb. Moreover, under such conditions, rule-of-thumb behavior may actually be optimal, in that it may satisfy Akerlof and Yellen's (1985) concept of near rationality. The analysis performed in this section is suggestive, however, and only looks at the resulting behavior of each type of agent; neither the costs of becoming a sophisticated forecaster nor the potential utility loss from forecasting with rules-of-thumb are modeled explicitly.

The left panel of Figure 1 plots the simulated output paths for representative sophisticated and rule-of-thumb agents using the same parameter settings as in Table 2-B. As shown in this panel, despite significant differences in the way they form expectations, sophisticated and rule-of-thumb forecasts behave in an almost identical manner. In particular, as sophisticated agents anticipate and react to the imperfections embedded in the forecasting schemes of rule-of-thumb agents, they effectively end up mimicking their actions. In the presence of complementarities, it pays to produce more [less] whenever aggregate output is higher [lower], even if the rise [decline] in output is largely due to the suboptimal forecasts of rule-of-thumb agents (and not warranted by true fundamentals). The right panel of Figure 1 shows that even in their investment decisions, which correspond to much more volatile series, the actions of sophisticated and rule-of-thumb agents are remarkably similar.

Figure 1 suggests that the potential utility losses from being a rule-of-thumb forecaster are likely small, implying that this course of action might well constitute a near-rational strategy. Thus, the aggregate effects of rule-of-thumb forecasters cannot be explained by simply looking at their actions in isolation; a more interesting and important factor lies in the endogenous response that their actions elicit from the sophisticated forecasters, a phenomenon that was also shown to play an important role in the model's persistence generation mechanism.

\section{$5 \quad$ Summary and Concluding Remarks}

The model described in this paper suggests strong quantitative effects at the aggregate level from allowing even a minority of agents to form expectations according to simple rules of thumb. Indeed, combined with strategic complementarity, forecast heterogeneity can strengthen the internal 
propagation mechanism of the model, as hypothesized by Haltiwanger and Waldman (1989) and Oh and Waldman (1994). The intuition is straightforward: As sophisticated agents try to forecast the forecasts (and actions) of others, they effectively end up reinforcing the perceptions of less sophisticated forecasters, even if these perceptions are not entirely consistent with the structure of the economy. This occurs because the sophisticated agents ultimately care about the aggregate state of the economy, and thus they will produce more whenever gains in aggregate output are foreseen, regardless of whether these gains are a result of the unsophisticated forecasts of the rule-of-thumb agents.

The above findings are quantitatively relevant only if the degree of strategic complementarity is sufficiently high, and there is still much uncertainty surrounding the empirical measures of the complementarity parameter at the aggregate level. Indeed, while the work of Basu and Fernald $(1995,1997)$ favors a low value for the strategic complementarity parameter, many of the (sectoral) estimates obtained by Cooper and Haltiwanger (1996) would place $\phi$ well above the range of values analyzed in this paper. Nonetheless, apart from concerns related to the magnitude of $\phi$, this paper can be interpreted as a relatively conservative approach to the analysis of the economic implications of forecast heterogeneity: After all, the rule-of-thumb forecasters depicted herein are still highly sophisticated individuals. In particular, they are smart enough to correctly solve their dynamic optimization problem and sufficiently well informed to know the exact nature of the stochastic process for technology shocks and to observe the contemporaneous actions of all the agents in the economy. ${ }^{5}$ Indeed, given how much the rule-of-thumb agents are allowed to know, one might even be surprised as to the extent to which such a small limitation in their behavior mattered as much as it did for any degree of strategic complementarity.

\footnotetext{
${ }^{5}$ Krusell and Smith (1996) analyze an artificial economy where agents are allowed to adopt simple savings rules of thumb instead of basing their savings behavior on the solution of a standard dynamic optimization problem. The time series properties of the rule-of-thumb economy are substantially different from those of a traditional artificial economy.
} 


\section{References}

Akerlof, G. and J. Yellen, 1985, Can small deviations from rationality make significant differences to economic equilibria?, American Economic Review 75, 708-20.

Arthur, W. B., 1994, Inductive reasoning and bounded rationality, American Economic Review Papers and Proceedings 84, 406-411.

Basu, S. and J. Fernald, 1995, Are apparent productive spillovers a figment of specification error?, Journal of Monetary Economics 36, 165-188.

Basu, S. and J. Fernald, 1997, Returns to scale in U.S. production: Estimates and implications, Journal of Political Economy 105, 249-283.

Baxter, M. and R. King, 1991, Productive externalities and business cycles, Working paper, University of Rochester.

Board, R., 1994, Polynomially bounded rationality, Journal of Economic Theory 63, 246-270.

Caballero, R. J. and R. K. Lyons, 1989, The role of external economies in U.S. manufacturing, NBER Working paper No. 3033.

Caballero, R. J. and R. K. Lyons, 1992, External effects in U.S. Procyclical Productivity, Journal of Monetary Economics 29, 209-225.

Cogley, T. and J. Nason, 1995, Output dynamics in real-business-cycle models, American Economic Review 85, 492-511.

Cooper, R. and A. John, 1988, Coordinating coordination failures in Keynesian models, Quarterly Journal of Economics 103, 441-63.

Cooper, R. and J. Haltiwanger, 1996, Evidence on macroeconomic complementarities, 1996, Review of Economics and Statistics 78, 78-93.

DeLong, J. B., A. Shleifer, L. Summers and R. Waldmann, 1990, Noise trader risk in financial markets, Journal of Political Economy 98, 703-738.

Evans, G. and G. Ramey, 1992, Expectation calculation and macroeconomic dynamics, American Economic Review 82, 207-224.

Frydman, R. and E. Phelps, 1983, Individual forecasting and aggregate outcomes:'Rational expectations' examined (Cambridge University Press).

Haltiwanger, J. and M. Waldman, 1985, Rational expectations and the limits of rationality: An analysis of heterogeneity, American Economic Review 85, 326-340. 
Haltiwanger, J. and M. Waldman, 1989, Limited rationality and strategic complements: The implications for macroeconomics, Quarterly Journal of Economics 104, 463-483.

King, R. G., 1995, Quantitative theory and econometrics, Federal Reserve Bank of Richmond Economic Quarterly 81, 53-105.

King, R. G., C. Plosser and S. Rebelo, 1990, Production, growth and business cycles: Technical appendix, Working paper, Rochester Center for Economic Research, University of Rochester.

King, R. G., C. Plosser and S. Rebelo, 1988, Production, growth and business cycles: 1. The Basic Neoclassical Model, Journal of Monetary Economics 21, 195-232.

Krusell, P. and A. Smith, 1996, Rules of thumb in macroeconomic equilibrium: A quantitative analysis, Journal of Economic Dynamics and Control 20, 527-558.

Kydland, F. and E. Prescott, 1982, Time to build and aggregate fluctuations, Econometrica 50, $1345-1370$.

Kydland, F. and E. Prescott, 1996, The computational experiment: An econometric tool, Journal of Economic Perspectives 10, 69-85.

Lucas, R. E., 1975, An equilibrium model of the business cycle, Journal of Political Economy 83, $1113-1144$.

Oh, S. and M. Waldman, 1994, Strategic complementarity slows macroeconomic adjustment to temporary shocks, Economic Inquiry 32, 318-329.

Roll, R., 1997, Nominal interest rates and loan volume with heterogeneous beliefs, 1997, Financial Markets, Institutions and Instruments 6, 1-58.

Sargent, T., 1993, Bounded rationality in macroeconomics (Clarendon, Oxford, UK).

Sethi, R. and R. Franke, 1995, Behavioural heterogeneity under evolutionary pressure: Macroeconomic implications of costly optimization, Economic Journal 105, 583-600.

Townsend, R., 1983, Forecasting the forecast of others, Journal of Political Economy 91, 546-588. 
Table 1 - Expectational Heterogeneity and the Propagation Mechanism ${ }^{a}$ Effect of Alternative Expectational Assumptions on the Autocorrelation of Aggregate Output

\begin{tabular}{|c|c|c|c|c|c|c|c|c|c|}
\hline \multicolumn{10}{|c|}{ A. Model without Strategic Complementarity $(\phi=0, \rho=0.5)$} \\
\hline & \multicolumn{3}{|c|}{$\alpha=0.3$} & \multicolumn{3}{|c|}{$\alpha=0.5$} & \multicolumn{3}{|c|}{$\alpha=0.7$} \\
\hline & auto(1) & $\operatorname{auto}(2)$ & auto(3) & $\operatorname{auto}(1)$ & $\operatorname{auto}(2)$ & auto(3) & $\operatorname{auto}(1)$ & $\operatorname{auto}(2)$ & $\operatorname{auto}(3)$ \\
\hline$\theta_{R}=0.0$ & 0.70 & 0.38 & 0.22 & 0.70 & 0.38 & 0.22 & 0.70 & 0.38 & 0.22 \\
\hline$\theta_{R}=0.3$ & 0.68 & 0.37 & 0.21 & 0.70 & 0.38 & 0.22 & 0.71 & 0.39 & 0.22 \\
\hline$\theta_{R}=0.6$ & 0.67 & 0.36 & 0.20 & 0.70 & 0.38 & 0.22 & 0.72 & 0.39 & 0.23 \\
\hline$\theta_{R}=0.9$ & 0.65 & 0.34 & 0.20 & 0.70 & 0.38 & 0.22 & 0.73 & 0.40 & 0.24 \\
\hline \multicolumn{10}{|c|}{ B. Model with Strategic Complementarity $(\phi=0.49, \rho=0.5)$} \\
\hline & \multicolumn{3}{|c|}{$\alpha=0.3$} & \multicolumn{3}{|c|}{$\alpha=0.5$} & \multicolumn{3}{|c|}{$\alpha=0.7$} \\
\hline & auto(1) & $\operatorname{auto}(2)$ & auto(3) & $\operatorname{auto}(1)$ & auto(2) & auto(3) & auto(1) & auto(2) & $\operatorname{auto}(3)$ \\
\hline$\theta_{R}=0.0$ & 0.84 & 0.65 & 0.55 & 0.84 & 0.65 & 0.55 & 0.84 & 0.65 & 0.55 \\
\hline$\theta_{R}=0.3$ & 0.86 & 0.63 & 0.49 & 0.89 & 0.71 & 0.59 & 0.92 & 0.77 & 0.67 \\
\hline$\theta_{R}=0.6$ & 0.85 & 0.62 & 0.47 & 0.91 & 0.75 & 0.65 & 0.95 & 0.87 & 0.81 \\
\hline$\theta_{R}=0.9$ & 0.87 & 0.65 & 0.53 & 0.92 & 0.78 & 0.70 & 0.94 & 0.86 & 0.81 \\
\hline
\end{tabular}

${ }^{a}$ Each expectational assumption is defined by a $\left(\alpha, \theta_{R}\right)$ pair. $\alpha$ is the perceived value of $\rho$ - the true AR(1) coefficient of the technology shock - and $\theta_{R}$ is the proportion of rule-of-thumb forecasters in the population. The table entries show the first 3 autocorrelations of aggregate output for different combinations of $\alpha$ and $\theta_{R}$. All standard RBC parameters are calibrated as in King et al. (1988). 
Table 2 - Comparing Selected Moments ${ }^{a}$

\begin{tabular}{|c|c|c|c|c|c|}
\hline Series & Std Dev & Rat. SD & auto $(1)$ & $\operatorname{auto}(2)$ & auto $(3)$ \\
\hline \multicolumn{6}{|c|}{ A. U.S. Postwar Quarterly Data ${ }^{b}$} \\
\hline Output & 5.62 & 1.00 & .96 & .91 & .85 \\
\hline Consumption & 3.86 & 0.69 & .98 & .95 & .93 \\
\hline Investment & 7.61 & 1.35 & .93 & .78 & .62 \\
\hline Hours & 2.97 & 0.52 & .94 & .85 & .74 \\
\hline \multicolumn{6}{|c|}{ B. Model with S.C. and Heterogeneous Expectations ${ }^{c}$} \\
\hline Output & 5.62 & 1.00 & .97 & .91 & .86 \\
\hline Consumption & 4.73 & 0.84 & .96 & .95 & .95 \\
\hline Investment & 10.96 & 1.95 & .85 & .68 & .54 \\
\hline Hours & 1.89 & 0.34 & .81 & .59 & .42 \\
\hline Prdvty Shock & 0.53 & 0.09 & .70 & .49 & .34 \\
\hline \multicolumn{6}{|c|}{ C. Standard RBC Model ${ }^{d}$} \\
\hline Output & 5.62 & 1.00 & .85 & .63 & .47 \\
\hline Consumption & 4.46 & 0.79 & .16 & .21 & .25 \\
\hline Investment & 16.24 & 2.89 & .67 & .44 & .29 \\
\hline Hours & 3.19 & 0.57 & .66 & .43 & .26 \\
\hline Prdvty Shock & 3.85 & 0.69 & .70 & .49 & .34 \\
\hline
\end{tabular}

${ }^{a}$ The first column of numbers shows the standard deviation of each series; the second column shows ratios of standard deviations of each series with output. Columns 3 through 4 show first, second, and third autocorrelation coefficients.

${ }^{b}$ Source: King, Plosser, and Rebelo (1988).

${ }^{c} \theta_{R}=0.30, \phi=0.50, \alpha=\rho=0.70$. All other parameters calibrated as in King et al. (1988).

${ }^{d} \theta_{R}=0.00, \phi=0.00, \rho=0.70$. All other parameters calibrated as as in King et al. (1988). 
Figure 1

Comparing the behavior of Sophisticated and Rule-of-Thumb agents

Output

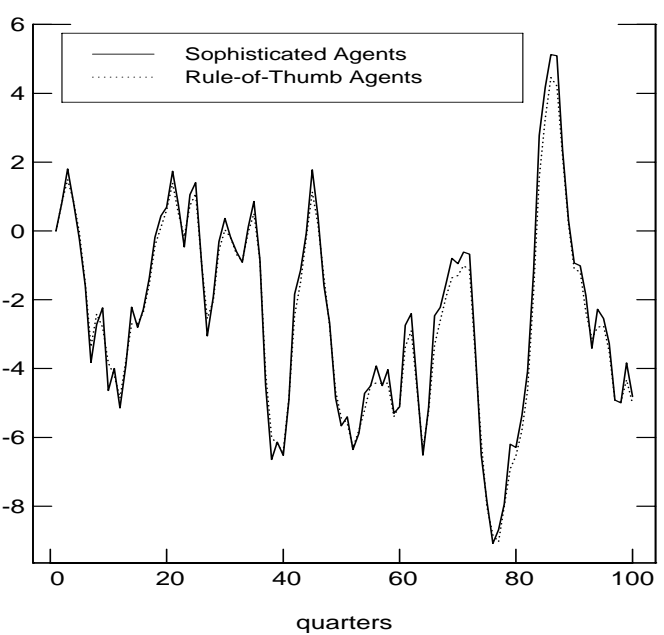

Investment

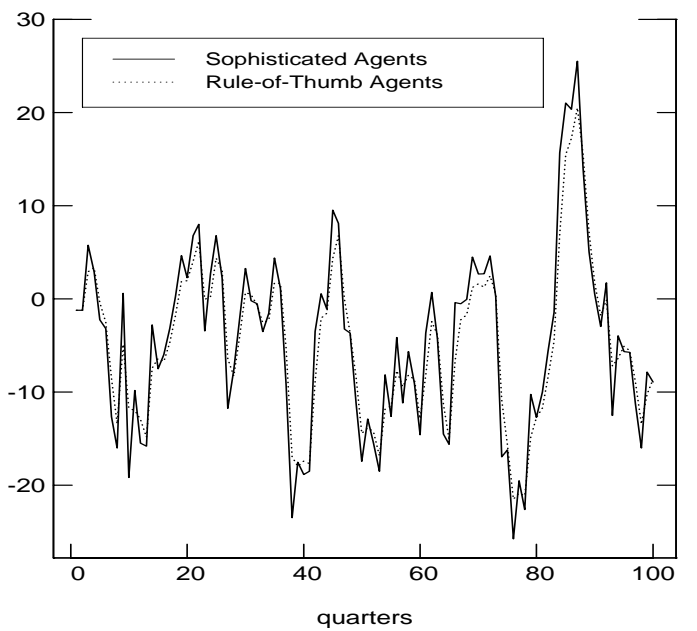

* The charts show the output and investment decisions of representative sophisticated and rule-of-thumb agents.

The parameterization is the same described for Table 2-B. $\left(\alpha=\rho=0.70, \phi=0.50, \theta_{R}=0.30\right)$ 